\title{
A golden opportunity for DNA detection
}

Unmodified gold nanoparticles can be used for simple and fast sequence specific detection of DNA.

Owing to the increasing use of sequence-specific DNA detection, there is a need to reduce its complexity, time and cost. Three

recent publications by Li and Rothberg demonstrate a new way to accomplish this. They observed that, under the proper conditions, single-stranded DNA (ssDNA) binds to unmodified gold nanoparticles (GNPs) but double-stranded DNA (dsDNA) does not (Li and Rothberg, 2004a,b). This difference has been used to develop assays using commercially available materials that are faster, simpler and cheaper than current methods.

In the presence of negative ions GNPs form a pink colloidal suspension. If the charge is screened by adding salt, the GNPs aggregate, turning the mixture blue. Li and Rothberg observed that ssDNA can bind to the particle surface and prevent this screening, whereas dsDNA cannot. Upon addition of a DNA mixture to the gold colloid, short ssDNA oligonucleotides immediately bind to the particles. Adding salt results in a color change that can be noticeably inhibited by a concentration of ssDNA just above that of the GNPs. Detection of ssDNA quantities as low as 60 femtomoles is possible by eye alone.

For sequence-specific detection of DNA in a sample such as that from a PCR reaction, short ssDNA probes are added to the sample; the mixture is melted and rehybridized under probe optimized conditions; and a tiny amount is added to the gold colloid. Adding salt turns the mixture bluish only if the probe had bound to the target. Long ssDNAs such as the target do not readily bind to the GNPs and do not interfere with this process. Careful control of the hybridization temperature enables single-nucleotide mismatches to be detected.
The sensitivity of the assay can be increased several orders of magnitude by exploiting a different property of the GNPs that enables them to quench a fluorescent tag on the probe (Li and Rothberg, 2004c). Although it requires additional equipment for detection, according to Rothberg this method has two advantages: "The first is that it is more sensitive, but what is more important is that it is mixture tolerant. For PCR this isn't important but [in] a complex mixture, as long as the tagged probes find something in the target, they won't be quenched. Further down the road, using the fluorescence method we can eliminate PCR altogether or do a lot fewer cycles."

Other methods use modified GNPs for detection of DNA and take much longer. Furthermore, Rothberg says, "We can make conditions perfect for hybridization because it occurs in a different tube. In other methods, conditions must be compatible with keeping the gold colloid stable. With this new method you hybridize anywhere under your favorite conditions and then add it to the gold colloid." This is likely to be just the beginning. As Rothberg says, "We've done a lot of applications [but] we've published just the tip of the iceberg."

\section{Daniel Evanko}

\section{RESEARCH PAPERS}

Li, H. \& Rothberg, L.J . Colorimetric detection of DNA sequences based on electrostatic interactions with unmodified gold nanoparticles. Proc. Natl. Acad. Sci. USA 101, 14036-14039 (2004a).

Li, H. \& Rothberg, L.J. Label-free colorimetric detection of specific sequences in genomic DNA amplified by the polymerase chain reaction. J. Am. Chem. Soc. 126, 10958-10961 (2004b).

Li, H. \& Rothberg, L.J. DNA sequence detection using selective fluorescence $q$ uenching of tagged oligonucleotide probes by gold nanoparticles. Anal. Chem. 76, 5414-5417 (2004c).

\section{CHEMICAL BIOLOGY}

\section{Proteins at the cross roads}

With a new cell-free assay, yeast proteins necessary for trafficking from the trans-Golgi network to the late endosome can easily be identified.

After proteins are synthesized, the cell's transport machinery shuttles them to their final site of action. Robert Fuller and his group at the University of Michigan have long been interested in a particular hub in the yeast cell, the trans-Golgi network (TGN). In a recent article in the Journal of Biological Chemistry, they describe a cell-free assay that they developed to hone in on the intersection between TGN and the late endosomal prevacuolar compartment (PVC), where proteins are sorted for recycling or degradation (Blanchette et al., 2004). Their goal was to biochemically identify the players in TGN to PVC trafficking.

A TGN membrane, bearing a TGN-specific protease, was mixed with a PVC membrane harboring a three component fusion protein consisting of (i) a protein whose role in TGN-to-PVC transfer was to be tested (ii) a reporter enzyme and (iii) a protease cleavage site followed by an epitope tag. If the protein of interest was involved in TGN-to-PVC membrane trafficking, it translocated the protease and the tag was subsequently cleaved. The efficacy of the transfer could then easily be quantified with the reporter enzyme. If the protein failed to transfer the protease, the fusion protein remained uncleaved and was removed by immunoprecipitation with the tag. Using this assay Fuller proved the essential role of the docking protein Pep $12 p$ for TGN-to-PVC trafficking and will now investigate other candidates such as clathrin or dynamin homologs.

"The advantage of this assay," Fuller says, "is that it is rapid, does not involve radioactivity or gels and is very quantitative." Fuller hopes to reconstruct the full mechanism in vitro, from TGN-vesicle budding to targeting and fusion with the late endosome-PVC vesicles. These findings will aid understanding of how the cell distinguishes between proteins targeted for recycling or degradation and those destined for secretion or localization elsewhere in the cell.

\section{Nicole Rusk}

\section{RESEARCH PAPERS}

Blanchette, J.M. et al. Cell-free reconstitution of transport form the trans-Golgi network to the late endosome/ prevacuolar compartment. J. Biol. Chem. published online 13 September 2004. 